\title{
BREVETS ET SOCIOLOGIE DU DROIT
}

Commentaire

Maurice Cassier

John Libbey Eurotext | « Sciences sociales et santé »

2008/4 Vol. 26 | pages 75 à 79

ISSN 0294-0337

Article disponible en ligne à l'adresse :

http://www.cairn.info/revue-sciences-sociales-et-sante-2008-4-page-75.htm

\section{Pour citer cet article :}

Maurice Cassier, «Brevets et sociologie du droit. Commentaire », Sciences sociales et santé 2008/4 (Vol. 26), p. 75-79.

DOI $10.3917 /$ sss.264.0075

Distribution électronique Cairn.info pour John Libbey Eurotext.

(C) John Libbey Eurotext. Tous droits réservés pour tous pays.

La reproduction ou représentation de cet article, notamment par photocopie, n'est autorisée que dans les limites des conditions générales d'utilisation du site ou, le cas échéant, des conditions générales de la licence souscrite par votre établissement. Toute autre reproduction ou représentation, en tout ou partie, sous quelque forme et de quelque manière que ce soit, est interdite sauf accord préalable et écrit de l'éditeur, en dehors des cas prévus par la législation en vigueur en France. Il est précisé que son stockage dans une base de données est également interdit. 
Sciences Sociales et Santé, Vol. 26, $n^{\circ} 4$, décembre 2008

\title{
Brevets et sociologie du droit
}

\author{
Commentaire
}

Maurice Cassier*

L'article de E. Cloatre sur " les brevets pharmaceutiques occidentaux et l'accès aux médicaments dans les pays pauvres : le cas de Djibouti face au droit international de la propriété intellectuelle » défend une posture originale : celle d'une sociologie du droit inspirée de la sociologie des sciences développée par B. Latour. Il s'agit d'évaluer les effets concrets des textes juridiques, en l'occurrence l'accord sur les Aspects de la propriété intellectuelle liés au commerce (ADPIC), qui est un texte fondateur de l'Organisation mondiale du commerce (OMC) en 1994, sur le marché des médicaments d'un petit pays (Djibouti) qui fait partie des pays les moins avancés. Comment les accords ADPIC sont-ils arrivés à Djibouti, par quels chemins, et quels sont leurs effets socioéconomiques? Vingt-cinq entretiens conduits auprès d'acteurs de la santé, du commerce et de l'industrie supportent cette analyse. L'arrivée du nouveau droit des brevets intéressant les médicaments paraît difficile en raison de l'absence d'institutions et d'expertise spécialisées dans le pays : "un membre » du ministère du Commerce est chargé de préparer la nouvelle loi, et cette loi reste introuvable au moment de l'enquête. L'article se plait à décrire des situations paradoxales: avant les accords de l'OMC, Djibouti ne disposait pas de loi sur les brevets de médicaments et, pourtant, le marché local organisé par les pharmaciens libéraux était dominé par les médicaments

\footnotetext{
* Maurice Cassier, sociologue, CERMES, Site CNRS, 7, rue Guy Môquet, 94801 Villejuif, France ; cassier@vjf.cnrs.fr
} 
brevetés et de marque importés. Après les accords de l'OMC, les génériques arrivent, portés par les politiques d'accès aux médicaments de la Banque mondiale dans le contexte de l'épidémie de sida. Les génériques n'étaient pas présents quand il n'y avait pas de droit des brevets; et ils arrivent en même temps que l'accord international sur les brevets de médicament! L'existence et les effets des lois sur les brevets supposent une connexion entre des textes de droit et des acteurs ou des réseaux socioéconomiques singuliers et situés, qu'ils soient locaux ou internationaux.

L'idée que le droit, pour exister, suppose d'être mobilisé par des acteurs est une idée commune en sociologie du droit. C'est une idée émise par Weber dans Économie et société : "Quand on parle de droit, d'ordre juridique, de règle de droit, on doit être particulièrement attentif à distinguer les points de vue juridique et sociologique. Le juriste se demande ce qui a valeur de droit du point de vue des idées... Le sociologue se demande en revanche ce qu'il en advient en fait dans la communauté... » (Weber, 2003 : 11). Cette approche a été déclinée dans le domaine du droit du travail ou de l'environnement par Serverin et Lascoumes (1995) pour lesquels la règle de droit est mobilisée et actualisée dans des situations concrètes. Ainsi, si l'on fait la carte des systèmes de brevets de médicaments, on s'aperçoit bien vite que les pays qui disposent de droits de brevets forts sur les médicaments sont dotés d'une forte recherche et innovation pharmaceutiques, souvent construite dès le début du XXe siècle, qu'il s'agit de défendre et de rémunérer sur des marchés réservés par des droits de propriété étendus; que les pays qui revendiquent des droits plus faibles entendent construire et défendre une industrie de génériques fondée sur la copie (Inde, Brésil) ; que les pays sans industrie, sans recherche et sans marché comme Djibouti ne disposaient pas, jusqu'à récemment, de système de brevet. Si le droit des brevets informe l'économie (Foucault, 2004), il est aussi informé et produit par les acteurs et le système économiques. Il ne faut donc pas s'étonner que les lois et les débats sur les brevets soient peu présents à Djibouti où le contrôle du marché pharmaceutique n'est pas un enjeu économique très disputé. Pour autant, et cela n'est pas abordé par l'article, les pays les moins avancés à l'instar de Djibouti vont subir très fortement les effets des accords de l'OMC sur les brevets de médicament dans la mesure où les possibilités de copie se ferment, y compris en Inde et en Chine, et qu'il deviendra plus coûteux d'importer les nouveaux médicaments brevetés dès lors que le marché des génériques s'étiole. Les brevets font sentir leurs effets même si le pays en question n'a pas encore de loi de propriété industrielle. Les marchés extérieurs sont désormais structurés par des brevets de produits pharmaceutiques qui sont étendus à l'échelle inter- 
nationale. Simultanément, et l'article précité le souligne fort justement, ces droits de brevets plus forts sont associés à des revendications d'accès aux traitements dans le contexte de l'épidémie de sida. $L$ ' "association" entre génériques et sida est donc singulièrement forte et elle touche aussi Djibouti. Elle a notamment été construite en Afrique du Sud et au Brésil par l'action conjointe des États, des associations de malades et des industriels producteurs de génériques. Elle a été produite par la politique de distribution gratuite des médicaments génériques mise en place par l'État brésilien à la fin des années 1990 et par le procès de Prétoria en Afrique du sud en 2001 (Cassier, 2008).

La sociologie du droit peut également s'intéresser aux institutions et aux acteurs qui fabriquent le droit dans le cours de leur action quotidienne. Il existe ainsi des monographies détaillées sur le travail des examinateurs de brevets de médicament au Brésil qui reposent sur des observations et des entretiens dans les groupes de travail des examinateurs et des juristes qui les assistent (1). Cette sociologie-là s'inspire du travail de Latour sur la fabrique du droit au Conseil d'État en France (Latour, 2002). L'avantage d'une observation à cette échelle est de montrer le travail d'interprétation de la règle de droit (par exemple, comment interpréter la règle de nouveauté dans le droit des brevets) et de suivre les discussions et l'élaboration des guidelines pour le travail des examinateurs des brevets. On pourrait croire que ce travail minutieux et extrêmement local est éloigné des débats internationaux sur les brevets de médicament. Il n'en est rien. Par exemple, les examinateurs brésiliens observés ont élaboré des règles qui concluent que le deuxième usage d'une molécule pharmaceutique déjà connue est non brevetable. C'est une énorme controverse avec les laboratoires internationaux. Dans un autre contexte, l'historien D. Kevles a suivi pas à pas les discussions entre les juristes et les scientifiques de la General Electric qui ont construit le brevet sur la bactérie Chakrabarty dont la brevetabilité fut tranchée par la Cour suprême des États-Unis par 5 voix pour et 4 voix contre (Kevles, 2002). L'historien G. Galvez-Béhar (2004) s'est quant à lui intéressé au travail des conseils en propriété industrielle dans la fabrication du droit des brevets en France à la fin du XIXe et au début du XXe siècles. Nous nous sommes intéressés, pour notre part, au travail d'un comité hybride de l'Office des brevets français, composé d'industriels, de scientifiques et de

(1) Voir le récent mémoire de master soutenu en avril 2008 à l'Université de l'État de Rio-de-Janeiro par E. Ribas de Biase Guimaraes : "Droit à la santé et propriété intellectuelle des médicaments au Brésil. L'approbation préalable de l'Agence nationale de surveillance sanitaire », Université de l'État de Rio-de-Janeiro. 
juristes, qui a changé dans l'entre-deux guerres les normes de brevetabilité des inventions pharmaceutiques en France avant que la loi ne change elle-même en 1944 (Cassier, 2008). Ces travaux se proposent de suivre la fabrication concrète du droit en observant le travail des examinateurs de brevets, des ingénieurs brevets, des industriels et des scientifiques qui mettent en forme les brevets et qui sont susceptibles de déplacer la règle de droit (2).

On peut aussi saisir la fabrication du droit des brevets en étudiant des controverses, des procès ou des oppositions juridiques au brevet. Kevles (2002) a ainsi étudié le procès du brevet de la bactérie Chakrabarty à la fin des années 1970 aux États-Unis. Nous avons, pour notre part, analysé les oppositions citoyennes aux brevets européens dans le domaine du vivant et des biotechnologies depuis le début des années 1990 (Cassier et Stoppa-Lyonnet, 2005). E. de Biase entend analyser au Brésil un procès encore méconnu qui porte sur l'annulation du brevet sur le Taxotère ${ }^{\circledR}$ par les examinateurs de l'Agence du médicament brésilienne, l'ANVISA. On peut encore esquisser l'étude du procès sur le Glivec $^{\circledR}$ qui opposa, en Inde, le propriétaire de la molécule, Novartis, à une alliance originale entre une association de malades du cancer et des industriels "génériqueurs ». La sociologie du droit doit ici sortir du cercle des juristes spécialisés et des offices de brevets pour s'intéresser aux associations de malades, aux Organisations non gouvernementales $(O N G)$, qui, le cas échéant, s'allient à des industriels du générique pour contester des brevets. Les brevets de médicament sont devenus un objet public et politique en même temps qu'ils sont un enjeu industriel. Les ONG intègrent désormais une contre-expertise juridique pour déposer des dossiers d'opposition ou de procès. Au Brésil, une opposition déposée par un laboratoire pharmaceutique public, propriétaire du ministère de la Santé, et une association de malades, ABIA, a récemment débouché sur l'annulation des brevets sur un antirétroviral, le ténofovir. La sociologie $d u$ droit des brevets de médicament rend ainsi lisible la construction des nouveaux rapports de force au sein de l'économie pharmaceutique mondiale, entre Etats, ONG, génériqueurs et laboratoires propriétaires, ces épreuves juridiques étant des moments de la construction et de l'agencement des forces socioéconomiques.

(2) Voir également Cambrosio et Keating (1996). 


\section{RÉFÉRENCES BIBLIOGRAPHIQUES}

Cambrosio A., Keating P., 1996, Technique, outil, invention : les transformations d'une biotechnologie, Sociologie du Travail, 38, 349-364.

Cassier M., Stoppa Lyonnet D., 2005, L'opposition contre les brevets de Myriad genetics et leur révocation totale ou partielle en Europe : premiers enseignements, médecine/sciences, 21, 658-662.

Cassier M., 2008, Patents and public health in France: exclusion of drug patents and granting of pharmaceutical process patents between the two World Wars, History and Technology, 24, 2, 135-151.

Cassier M., 2008, Géopolitique du médicament, In : Zilberman P., ed., Épidémies, Paris, Éditions de la Martinière, sous presse.

Foucault M., 2004, Naissance de la biopolitique, Paris, Le Seuil.

Galvez-Béhar G., 2004, Pour la fortune et pour la gloire. Inventeurs, propriété industrielle et organisation de l'invention en France, 1870-1922, thèse Université Lille-III.

Kevles D., 2002, A history of patenting life in the United States. With comparative attention to Europe and Canada, Report to the European Group of Ethics.

Latour B., 2002, La fabrique du droit. Une ethnographie du Conseil d'État. Paris, La Découverte.

Serverin E., Lascoumes P., 1995, Le droit comme activité sociale : pour une approche wébérienne des activités juridiques, Droit et Société, 1, 171-193

Weber M., 2003, Économie et société, tome 2, Les relations fondamentales entre l'économie et l'organisation sociale, Paris, Agora. 